\title{
Die Chamberlens.
}

\author{
Geschichtliche Skizze nach J. H. A veling.
}

Von

\author{
Dr. II. Sänger, \\ Privatdocent in Leipzig.
}

(Mit 6 Holzschnitten.)

Der deutschen Geburtshülfe, welche den Erzeugnissen der ausländischen Fachliteratur allzeit gebührende Aufmerksamkeit zuwendet, ist bisher ein Werk entgangen, dessen Inhalt in hohem Grade verdient, überall bekannt und gewürdigt zu werden, wo man für die geschichtliche Seite unseres Faches noch ein Interesse hegt. Es ist das Buch des hochangesehenen Londoner Geburtshelfers J. H. Aveling ${ }^{1}$ ) über die Chamberlens und die Urgeschichte der Geburtszange. Diese Arbeit, eine Frucht zehnjähriger archivalischer Studien, führte zu dem Ergebniss, dass die geschichtlichen Angaben, welche seither über die Chamberlens gemacht worden sind, falsche waren, sofern keines von den Mitgliedern der Familie, dem bisher, der Reihe nach, die Erfindung der Zange zugesprochen wurde, auf diese Ehre Anspruch hat. Weiterhin hat Aveling zum ersten Male den Lebensgang der verschiedenen Glieder der Chamberlen'schen Familie quellenmässig verfolgt und ist dadurch in den Stand gesetzt worden, das seither darüber Bekannte in ungeahnter Weise zu klären und zu vervollständigen.

Weder in dem Lehrbuche von Spiegelberg (2. Auflage), noch dem von Schröder (9. Auflage), noch auch in dem soeben

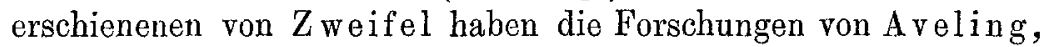
rein aus Unbekanntschaft mit denselben, Aufnahme gefunden. Es erschien mir daher als ein dankbares Unternehmen, den Inhalt des A veling'schen Buches, als eines auch vom allgemein medicinal- und culturgeschichtlichen Gesichtspunkte hochinteressanten

1) The Chamberlens and the Midwifery Forceps. Memorials of the Family and an Essay on the invention of the Instrument. London 1882. Churchill. 
Werkes, für den deutschen Leserkreis in gedrängtem Abrisse wiederzugeben. Ich muss aber ausdrücklich hervorheben, dass ich keineswegs eine blosse Uebersetzung biete. Wer diese Skizze mit dem Originale vergleichen will, wird erkennen, dass ich mich bemüht habe, die bei Aveling chronikartig lose aneinander gereihten Daten zu einem mehr abgerundeten Ganzen zu verbinden, zum besseren Verständuiss nöthige Erläuterungen einzuflechten und die Sprache meiner Schilderung zu einer mehr erzählenden als aufzählenden zu gestalten. Nur für den Schlusstheil des Buches, worin Aveling selbst als Essayist auftritt, habe ich, um seine Beweisführung genau wiedergeben zu können, mich treu an seine Darstellung gehalten.

Ich bringe zunächst den Stam mbaum der englischen Chamberlens unter Weglassung derjenigen Sprossen der Familie, welche geschichtlich nicht weiter in Betracht kommen.

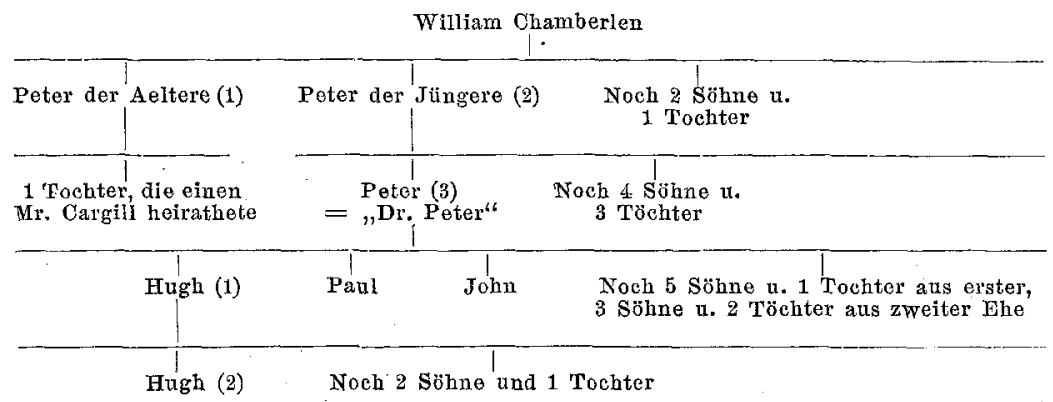

Mit $\mathrm{Hugh}$ (2), welcher drei Töchter hinterliess, erlischt der Mannsstamm der Chamberlens.

Beim Ueberblicken der Stammtafel fällt sogleich auf, dass zwei von den vier Söhnen William Chamberlen's den Vornamen Peter trugen, sowie dass der eine Sohn von Peter dem Jüngeren gleichfalls Peter hiess. Die seltsame Vorliebe für diesen Vornamen trug wesentlich dazu bei, die Nachforschungen nach den Leistungen jedes dieser drei Peters hinsichtlich der zu lösenden Frage zu erschweren. Auch Peter (3) und Hugh (1) gaben je einem ihrer Söhne den Vornamen Peter, welche sich aber zum. Glïck in keiner die Forschung erschwerenden. Weise hervorthaten.

Was die Schreibart des Familiennamens anlangt, so liess sich William Chamberlen in das Register der französischen Kirche zu Southhampton ,Chambrelein " eintragen. Von späteren Einträgen findet sich noch häufiger "Chambellan" (siehe 
unten). Seine beiden Söhne schrieben sich „Chamberlan“. Peter (3) anglisirte den ursprünglichen Namen weiter in ,Chamberlen", der von den folgenden Generationen unverändert beibehalten wurde.

William Chambellan lebte zur Zeit der Hugenottenkriege in Paris, wahrscheinlich als Arzt. Gleich zahlreichen anderen Protestanten wanderte er mit seinem Weibe Geneviève Vingn on nach England aus und liess sich in Southhampton nieder. Dies geschah im Jahre 1569, in welchem die Bedrückung der Hugenotten infolge mehrerer Niederlagen ihrer Führer Condé und Coligny eine besonders schwere war. Wäre William $\mathrm{Ch}$. in Paris geblieben, so würde es wohl auch ihm in der Bartholomäusnacht (14. August 1572) ans Leben gegangen sein.

Gemäss dem unter den Flüchtlingen herrschenden Brauche, sogleich nach der Ankunft zum Abendmahl zu gehen, was dann unter Anführung des Namens in dem noch vorhandenen Kirchenbuche verzeichnet wurde, ist uns dieser erste Eintrag erhalten worden. Er lautet: „Ensuyt les noms de ceux qui ont faict profession de leur foy et admis à la Cène. Chambrelein Villame reçu à la cène, 3. Jouillet $1569 . “ "$

Wenn auch nicht sicher bewiesen, so ist es doch in hohem Grade wahrscheinlich, dass William $\mathrm{Ch}$. als Arzt prakticirt habe. Diese Annahme lässt sich durch vier Gründe stützen: Zur damaligen Zeit war es in Frankreich protestantischen Aerzten nicht erlaubt, ihren Beruf auszuïben unter dem Vorwande, dass sie katholische Kranke nicht anwiesen, wenn es Zeit sei, die. Sacramente zu empfangen. Zweitens waren unter den Angehörigen der französischen Kirche in Southhampton auffallend viele.Aerzte. Drittens waren zwei seiner Söhne ebenfalls Aerzte; endlich rühmt Dr. Peter $\mathrm{Ch}$. von sich, dass er einer Asklepiaden-Familie angehöre und ,, von der Wiege auf" mit Arzneikunde vertraut gemacht worden sei.

Sonst ist über den Lebensgang von William Ch., den Stammvater der Familie in England, nichts bekannt. Sein Tod muss vor 1596 erfolgt sein, da er in einem die Geburt seines Sohnes Peter des Jüngeren betreffenden Documente aus diesem Jahre als bereits verstorben angeführt ist.

Es scheint, wenigstens bis auf Dr. Peter Ch. (William Ch.'s Enkel) sich die Tradition in der Familie erhalten zu haben, dass sje ursprünglich dem normannischen Adel zugehört habe und 
aus Tankervile (jetzt Tancarville) stamme. Dr. Peter Ch. verwahrt sich zwar in einer seiner Schriften gegen diese Abstammung, weil ihm daran gelegen war, als in London bürgerlich geboren zu gelten, aber er bediente sich, ebenso wie seine Vorfahren und Nachkommen, eines Wappens, welches auch das der normannischen Familie sein soll: auf rothem Grunde ein silbernes Wappenschild, umgeben von einem Kranze goldener Rosetten und darüber ein Balken mit drei aufrechten Sparren. $\left.{ }^{1}\right)$

Peter Ch. der Aeltere, der älteste Sohn von William Ch., ist um das Jahr 1560 noch in Paris geboren. Er siedelte zwischen 1588 und 1596 von Southhampton nach London über, um dort als Chirurg und Geburtshelfer zu prakticiren. Als Mitglied der „Barber Surgeons Company“ wird sein Name in deren Annalen zum ersten Male 1598 erwähnt.

Mehrfache Conflicte mit dem ,Royal College of Physicians“ wegen Ueberschreitung seiner Befugnisse, „de praxi illicita et mala" (Aerzte durften Chirurgie betreiben, Chirurgen aber keine inneren Krankheiten behandeln), führten endlich sogar zu seiner Gefangensetzung. Auf Vermittelung der Königin Anna (Gemahlin von Jacob I.) und des Erzbischofs von Canterbury wurde er dann wieder aus der Haft befreit. Peter Ch. hatte die Königin mehrmals entbunden, wofür er ausser dem Honorar (40 Pf. Sterl.) auch einen Diamantring bekam, dessen er in seinem noch erhaltenen Testamente erwähnt. Er starb 1631 als Wittwer, nachdem or 62 Jahre in England gelebt hatte. Von seinem Ansehen zeugt auch der Umstand, dass er Chirurg der ostindischen Compagnie wurde und ein grosses Vermögen hinterliess.

Peter Chamberlen der Jüngere, der Bruder des vorigen, ist am 18. Februar 1572 in Southhampton geboren. Er kam später mit Letzterem nach London und wurde gleichfalls in die „Barber Surgeons Company" aufgenommen, in welcher er es zur Würde eines „Master“" brachte, für welche der Nachweis seiner

1) Budin, welcher laut Notiz in einer Monographie von Poullet (Des diverses espèces de Forceps, leurs avantages et inconvénients. Paris 1883. Baillière) hierüber weitere Nachforschungen anstellt, bestätigte diese Abstammung der Chamberlens, ,von dem kleinen Adel der Normandie“. Uebrigens ist Poullet entweder des Englischen nicht genügend mächtig oder hat A veling nur flüchtig gelesen, da er eine ganze Reihe von irrthümlichen Angaben macht, auf welche ich hier, da sein Buch als Quelle benutzt werden könnte, hinweisen will, ohne mich aber veranlasst zu sehen, sie auch zu corrigiren. 
in England erfolgten Geburt erforderlich war. Auch er hatte beständig Conflicte mit den Aerzten. In den Annalen der Chirurgie wird besonders erwähnt, dass er sich viel mit Geburtshülfe befasste. Auf seine und seines älteren Bruders Veranlassung geschah es auch, dass die Hebammon Londons bei der Regierung und den Aerzten darum einkamen, eine Corporation bilden zu dürfen. Zu einer von dem College of Physicians behufs Prüfung der Angelegenheit eingesetzten Commission wurde laut dem noch vorhandenen Protokoll der letzteren auch Peter $\mathrm{Ch}$. d. J. zugezogen, was aus folgendem bemerkenswerthen Passus desselben hervorgeht: „Peter Ch. d. J., welcher unverschämter Weise sich der Sache dieser Weiber angenommen hat, wurde die Frage vorgelegt, ob er nicht zugebe, dass ein Mitglied des College of Physicians einen Fall von schwieriger Geburt besser und richtiger beurtheilen könne, als irgend ein Geburtshelfer-Chirurg (obstetric surgeon), wenngleich er und sein Bruder sich brüsteten, sie und sonst Niemand, in diesen Dingen besonders geschickt zu sein."

Hierin haben wir die erste öffentliche Kundgebung über die Existenz des Chamberlen'schen Familiengeheimnisses zu erblicken. Was mag wohl Peter Ch. d. J. in seinem Inneren gedacht haben, da ihm diese höhnische Frage vorgelegt wurde? Warum zeigte er den Aerzten nicht, ,that he and his brother, and none others, excelled in these subjects?"

Uebrigens wurden die Hebammen vorerst abgewiesen, vielleicht gerade, weil die Chamberlens deren Sache zu der ihrigen gemacht hatten.

Im Jahre 1620 wird Peter Ch. d. J. zum letzten Male in den Annalen der Chirurgenzunft erwähnt, abermals in Betreff einer Ueberschreitung seiner Competenz. Er starb, 54 Jahre alt, 1626, also mehrere Jahre vor seinem älteren Bruder.

Dr. Peter Chamberlen (3), Sohn von Peter Ch. d. J. (2), ist geboren am 8. Mai 1601 (siehe Bild S. 124). Seine Taufurkunde ist noch in französischer Sprache ausgestellt. Was dem Vater und Onkel versagt war, sollte der Sohn und Neffe erreichen: die Stellung als Physician, den Titel Doctor. 1615 finden wir den jungen Peter $\mathrm{Cl}$. in Cambridge, später in Heidelberg und Padua, wo er 1619 promovirte. Er hat dann noch in Oxford und Cambridge weiter studirt. 1626 wurde er in das College of Physicians als Mitglied aufgenommen, zwei Jahre später stieg er zum Range eines Fellow derselben auf. Eine längere Reihe von Jahren 
widmete er sich nunmehr seiner Praxis, dio ihn auch mit dem Hofe in Berührung brachte, durch welchen sein Ruf bis nach Russland drang. Der Czar (Michael Feodorowitsch Romanow) schrieb eigenhändig an Charles I. und bat, Dr. Chamberlen zu veranlassen, als sein Leibarzt nach Moskau zu kommen. Dr. Ch. lehnte $a b$ und an seiner Stelle ging ein in England ausgebildeter Russe (finnischer Schwede?) Elmston dorthin.

Die von seinem Vater und Onkel von Anfang an in selbstsüchtiger Weise angeregte Angelegenheit der Verleihung von Cor-

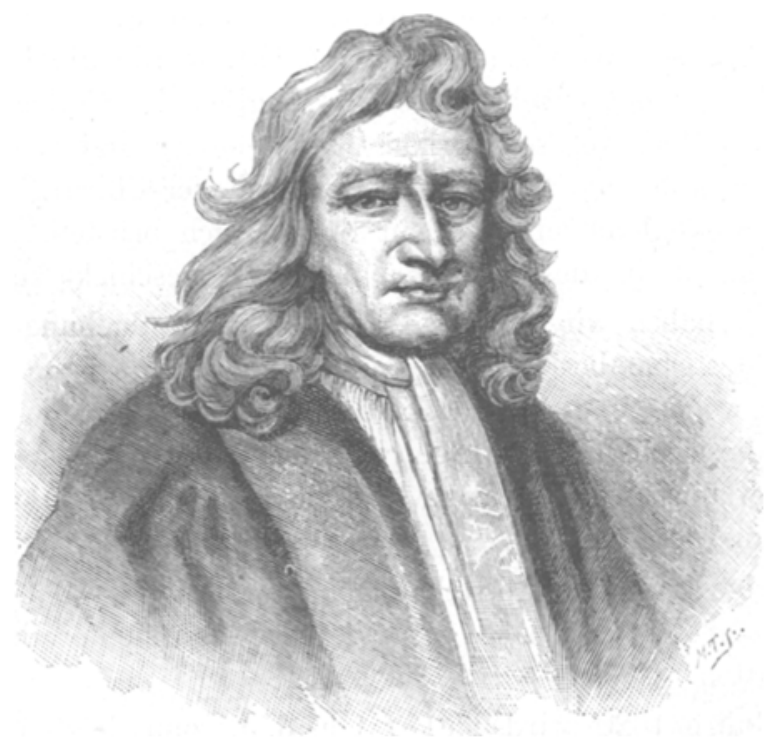

porationsrechten an die Hebammen suchte auch Dr. Peter Ch. in die Hand zu nehmen, verfeindete sich aber mit diesen durch seine anmaassenden und eigennützigen Forderungen, welche auf nichts Anderes hinausliefen, als dass er allein befugt sein sollte, die Hebammen zu unterrichten, zu prüfen, ihnen Diplome zu ertheilen. Sie richteten daher an das College of Physicians eine scharfe Petition gegen Dr. Peter Ch., welche am 18. Juni 1634 in seiner Gegenwart zur Verlesung kam und dann, vermehrt um eine weitere, sein Vorgehen in der schroffsten Weise verurtheilende Petition des College selbst, an den Bischof von London, dessen Jurisdiction die männlichen und weiblichen Geburtshelfer unterstanden, weiter ging. Sowohl die Hebammen, wie die Mitglieder des Col- 
lege, also die nächsten Berufsgenossën Dr. Peter's, wehrten sich gegen die von ihm angeblich geplante Monopolisirung der geburtshülflichen Praxis. Auch in der Sache des Hebammenunterrichtes habe er nicht mehr Recht als irgend ein Mitglied des College.

„Wenn er sich bei seinen Entbindungen eiserner Instrumente bediene, so thäten Andere dies auch und könne daraus kein Grund für seine vorgebliche Ueberlegenheit abgeleitet werden. Ueberdies habe er sich geweigert, arme Frauen zu entbinden und reichen nicht eher seinen Beistand geliehen, bis seine Forderungen befriedigt worden seien u. s. w." Am 22. October 1634 erschienen darauf beide Parteien im erzbischöflichen Palais von Lambeth, und wurde Dr. Peter Ch. das von ihm geforderte Recht völlig abgesprochen und er streng ermahnt, seine Hülfe Arm wie Reich allzeit und uneigennützig darzubieten, bei Androhung schwerer Strafe im Falle des Zuwiderhandelns.

Wie kläglich steht Dr. Peter Ch. in dieser Angelegenheit vor uns! Er lässt alle Schmähungen seiner Collegen und der Hebammen über sich ergehen, er demüthigt sich vor den geistlichen Behörden, aber sein einträgliches Geheimniss verräth er nicht,

Erst nach 13 Jahren, 1647, erlässt er eine Rechtfertigungsschrift gegen seine Widersacher und Feindinnen mit dem seltsamen Titel. ${ }^{1}$ ): „Eine Stimme in Rhama, oder: Der Schrei von Weibern und Kindern im Echo des Mitleids, von Peter Chamberlen etc." Indem er seine Feinde für das Blut der Frauen und Kinder verantwortlich macht, das da fliesse ,,durch die Unwissenheit und Verwirrung der ausser Controle stehenden weiblichen Schiedsrichter über Leben und Tod", sucht er sich selbst von allen gegen ihn erhobenen Vorwürfen, besonders dem der Habsucht, zu reinigen und seine Handlungsweise als lediglich von edlen Motiven geleitet hinzustellen. Er erzählt seine Abstammung, doch mit besonderer Hervorhebung seines englischen Indigenates (siehe S. 122), seinen Studiengang, seine ärztliche und geburtshülfliche Thätigkeit; er rühmt seine nur auf bessere Belehrung und staatliche Beaufsichtigung der Hebammen gerichteten Bestrebungen, die von neidischen Feinden durchkreuzt würden. Darum möge ,der Schrei der misshandelten Weiber und Kinder

1) „A Voice in Rhama, or: The Crie of Women and Children. Echoed forth in the Compassions of Peter Chamberlen, Doctour in Physick, Fellow of the Colledge of London and one of his Majesties Physicians Extraordinary." 
so laut und jammervoll ertönen, bis sich Samariter fänden, die sich ihrer (den Hebammen gegenüber) annähmen."

Sicherlich ist der Kern dieser Bestrebungen ein guter und richtiger gewesen, damals wie später, aber Dr. Peter Ch. war bei seiner Hinterhältlichkeit, seiner Selbstsucht, seinem den Zeitgenossen in der Unkenntniss des Familiengeheimnisses unbegreiflichen Eigendünkel nicht der Mann, sich zum Anwalt der gemarterten Weiber und Kinder aufzuspielen, deren Schrei auch ihm in die Ohren gellen musste.

Ein solcher Mann, vermochte er es noch über sich auszurufen: ,Ich bin kein Pharisäer, ich kann mein Thun vor Gott und den Menschen rechtfertigen und erkenne mich nicht schuldig, mein Gewissen dem grossen Götzen Habsucht geopfert zu haben." " Nach einer solchen Aeusserung nimmt es nicht Wunder, ihn schliessen zu sehen: ,Ich wusch meine Hände, ich reinigte meine Seele. Gott segne in seiner Allmacht und seinem Erbarmen diese Schrift zu seinem Ruhme. Amen."

Dass Dr. Peter Ch. aber doch auch ron seinen Zeitgenossen hinsichtlich seines Charakters wohl durchschaut wurde, geht aus einer 1650 erschienenen anonymen Schrift hervor mit dem ihren Inhalt genügend verrathenden Titel 1): „Eine Antwort auf Dr. Peter Chamberlaine's scandalöse und lügenhafte Schriften.".

In der Folge bethätigte sich Dr. Peter Chamberlen's unruhiger Geist in einer seltsamen Mischung von verständigen Vorschlägen mit manchem überraschend genialen, seiner Zeit vorauseilenden Gedanken und einer nie rastenden Projectenmacherei in Dingen, welche seinem Berufe gänzlich fernlagen.

So wandte er sich in einem noch öfter vortretenden Streben nach gemeinnützigen Unternehmungen zunächst der Errichtung von öffentlichen Bädern und Badestuben zu. Das Oberhaus gewährte ihm 1649 hierzu Licenz auf die Dauer von 14 Jahren. Das Unterhaus bestätigte dieselbe, bestimmt durch Dr. Peter Ch.'s ebenso gelehrte wie hygienisch vortreffliche Begründung. Das College of Physicians, von einem behufs Ausführung des Projectes zusammengetretenen Comité um ein Gutachten angegangen, übte unter wirklich nichtigen Vorwänden, wobei die Missbräuche und Schädlichkeiten, welche bei dem Ge-

1) An answer to Dr. Peter Chamberlaine's Scandalous and False Papers, by Philolethes. 
brauche von Bädern vorkommen könnten, die Hauptrolle spielten, eine äusserst abfällige Kritik, was wieder eine lange gelehrte und schlagfertige Entgegnung des schriftgewandten Dr. Peter Ch. hervorrief. Diese seine Vertheidigungsschrift kann mit zu den ältesten und originellsten Abhandlungen der balneologischen Literatur gezählt werden.

Die fortgesetzte Opposition der Aerzte gegen sein hauptsächlich dadurch gescheitertes Unternehmen war Veranlassung, dass Dr. Peter Ch. fortan nicht mehr unter ihnen erschien. Verschiedenen Ladungen zur Theilnahme an den Zusammenkünften des College leistete er keine Folge, und so wurde er am 23. November 1649 seiner Mitgliedschaft für verlustig erklärt.

Es kümmerte ihn wenig. War er doch gerade damit beschäftigt, wieder eine Schrift voll hochfliegender Gedanken herauszugeben mit dem Titel: „Des armen Mannes Anwalt oder Englands Samariter." Dieser war er selbst, und er versprach nicht mehr und nicht weniger, als, ,alle Parteien zu versöhnen; alle Bedürfnisse für die Armen und die Soldaten zu beschaffen, den letzteren auch ihren rückständigen Sold; alle öffentlichen Schulden, alle Schulden der früheren Könige, Königinnen, Prinzen zu bezahlen u. s. w." Wir lernen hier Dr. Peter Ch. als einen communistischen Weltverbesserer und Utopisten kennen, der Thomas Morus an Phantasie nichts nachgiebt. Gleich allen seinen Schriften hat auch diese einen gewissen religiösen Hintergrund, wie er auch nie schliesst, ohne Gott zum Zengen seiner guten Absichten angerufen zu haben. So ist es psychologisch verständlich, dass er nun in eine Periode religiöser Schwärmerei trat, welche sich in zahlreichen Streitschriften und Disputationen mit Laien, Juden, Geistlichen Luft machte, sich vorzugsweise um Wesen und Wirkung der Taufe drehten und stellenweise ganz das Gepräge religiöser Verrücktheit trugen. Dann sehen wir ihn wieder Religion mit Politik verquicken in einer 1659 dem Parlamente eingesandten Schrift: ,Aufgaben gesetzgeberischer Gewalt", worin er in 78 ,Problemen" zeigen will, wie ,die Gesetze der Menschen mehr in Uebereinstimmung zu bringen seien mit denen Gottes".

Dazwischen vergisst er seine persönlichen Vortheile keineswegs. Als ihn Carl II. zu seinem ordentlichen Leibarzt ernannte, ohne ihm ein Decret zustellen zu lassen, richtete er ein Gesuch an den König (Februar 1661) mit der Bitte um officielle Bestäti- 
gung seines Titels, wobei er das fertige, nur zu unterschreibende Patent gleich beilegte. ${ }^{1}$ )

Ausser einem von Dr. Peter Ch. für einen staatsgefangenen Colonel Lydcote sub dato 28. März 1662 ausgestellten ärztlichen Zeugniss, einer religiösen Streitschrift, die 1662 erschien; hörte man einige Jahre nichts von ihm, bis er mit einem Male, 1665, in Holland auftauchte. Von hier schrieb er seinem Sohne $\mathrm{Hugh} \mathrm{Ch.,} \mathrm{dass} \mathrm{or} \mathrm{ihm} \mathrm{in} \mathrm{England} \mathrm{Patent} \mathrm{verschaffen} \mathrm{möge} \mathrm{für}$ seine neue Erfindung ,der Fortbewegung von Schiffen und Wagen mit Hülfe des Windes und gerade gegen den Wind". Da sein Sohn abgewiesen wurde, kam er selbst nach England zurück und setzte es durch, dass ihm das Parlament die Licenz, solche Fahrzeuge zu bauen, mit denen er sein. Project verwirklichen könne; auf 14 Jahre ertheilte. Da aber trotzdem die Unterstützung des Publikums ausblieb, so gab er selbst seine chimärische Idee, die ihn den Erfindern eines Perpetuum mobile anreihte, auf, um sich wieder einer ganz anderen Sache zuzuwenden, nämlich dem Problem einer Lautschrift der englischen Sprache, was ihm vom König und Parlament, wie üblich, auf 14 Jahre patentirt wurde (1672).

Es nimmt nicht Wunder, dass Dr. Peter Chamberlen's excentrisches Leben und phantastische Schriften eine Menge hämischer und spöttischer Angriffe herausforderten, wie dass er verrückt, dass er untergegangen, dass er ein Jude sei u.s. w., auf welche er dann bei seiner immer mehr gesteigerten Empfindlichkeit in der ihm eigenen, nun schon genugsam charakterisirten Manier antwortete. Die Titel der Erwiderungsschriften, welche or auf jene drei Vorwürfe hin, sehr bezeichnender Weise in Lapidarbuchstaben, drucken liess, lauten: „Des vernünftigen Mannes Rechtfertigung", „Non inventus, oder: Das verlorene und wieder aufgefundene Schaf“, „Die Söhne des 'Ostens". Das Gerede, ein Jude zu sein, kam wohl daher, dass er mit solchen häufig disputirte und den jüdischen Sabbath hielt.

Eines der letzten Projecte Dr. Peter Ch.'s - das aussichtsloseste von allen - war ein religiös-reformatorisches: „Die Ver-

1) Es lautet in seiner originellen, kaum übersetzbaren Fassung folgendermaassen: „Doctor Peter Chamberlen, Physitian to Our Royall Father and Mother, Ordinarily Attending Our Happy Births, And (therefore) One of Our First Servants: Is Our Servant and Physician in Ordinary." 
söhnung aller Kirchen." Seine Hoffnungen und Pläne hierzu sind in einer Anzahl von Briefen erhalten, welche er an die Erzbischöfe Sheldon und Sancroft gerichtet hatte.

Dr. Peter Chamberlen starb am 22. December 1683 im Alter von 82 Jahren in Woodham Mortimer Hall in der Nähe von Maldon in Essex. Er ist auch daselbst begraben. Sein Grabmal ist in stark verfallenem Zustande noch vorhanden.

Dieses Woodham Mortimer Hall, welches er von einem Sir Cranmer Harris erstand und welches bis zum Jahre 1715 der Chamberlen'schen Familie verblieb, ist dieselbe Besitzung, wo 1813 durch Zufall deren geburtshülfliche Instrumente aufgefunden wurden.

In seiner selbstverfassten Grabschrift rühmt er sich seiner zahlreichen Nachkommenschaft, seiner Stellung als Leibarzt von drei Königen und Königinnen von England, seiner Reisen durch die meisten Länder Europas, deren Sprachen er auch gesprochen habe. Als Jahr seiner Taufe nennt er 1648, woraus zu entnehmen, dass er Wiedertäufer war, und versäumte nicht, auch hier noch zu erwähnen, dass ex seit 32 Jahren den siebenten Tag als Sabbath gefeiert habe. Das Auto-Epitaph schliesst mit einem langen Gedichte, dessen erste zwei Verse also lauten:

,To tell his learning and his life to men

"Enough is said, by Here lyes Chamberlen."

Wie anders würde man diese stolzen Zeilen gelten lassen, wenn Dr. Peter Ch. der. Welt das offenbart hätte, was alle seine Projecte aufwog, - die Geburtszange. So wird man aber ohne Bewegung die kurze und beissende Charakteristik des Mannes hinnehmen, welche ein nicht dem ärztlichen Stande angehöriger Zeitgenosse von ihm gab:

„The Man-Midwife and a Notorious Phanatic."

Hugh (Hugo) Chamberlen der Aeltere, der seither bekannteste unter den Chamberlens, derjenige, welcher am meisten von sich reden machte, dessen Lebensgeschichte Aveling aber noch um viele bemerkenswerthe Züge bereichern konnte, ist wahrscheinlich 1630 als der älteste Sohn von Dr. Peter Ch. in London geboren. Wie und wo er erzogen wurde, wie er zu seinem Doctortitel kam, darüber sind Belege nicht aufzufinden gewesen. Ueberhaupt liegen bis zu seinem Auftauchen in Paris (1670) nur spärliche und lückenhafte Nachrichten über seinen Lebensgang vor. Das erste Mal geschieht seiner quellenmässig Erwähnung in 
dem Journal des Oberhauses (1660), worin verzeichnet steht, dass ihm zu verschiedenen Gutskäufen und -Verkäufen Erlaubniss ertheilt wurde.

1663 verheirathete er sich mit der Tochter eines Obersten, John Brett, aus welcher Ehe drei Söhne, Hugh (der Jüngere), Peter (dor vierte) und Middleton, sowie eine Tochter entsprangen.

Die vom Vater vererbte Projectmacherei, welche, wie wir noch sehen werden, $\mathrm{Hugh} \mathrm{Ch}$. d. Ae. überkommen war, machte sich zum ersten Male bemerklich durch sehr allgemein gehaltene Vorschläge zur Befreiung der City von der Pest (1666). Nun hören wir nichts weiter von $\mathrm{Hugh} \mathrm{Ch}$. d. Ae. bis 1670 , wo er in Paris erschien und der Held jener berühmten Episode wurde, welche Mauriceau in seinen, ,Observations sur la grossesse et l'accouchement" geschildert hat und zu allgemein bekannt ist, um hier wiederholt $\mathrm{zu}$ werden. Ebensowenig brauche ich hier auf die berühmte Vorrede zu $\mathrm{Hugh} \mathrm{Ch.'s} \mathrm{Uebersetzung} \mathrm{des}$ Werkes von Mauriceau (1672) einzugehen, welche in ihren auf die Erfindung der Zange bezüglichen Anspielungen bis zu ihrer wahren Aufklärung zahllose Commentare und Deutungen hervorgerufen hatte.

Die englische Ausgabe des $\mathrm{Mauriceau}$ von $\mathrm{Hugh} \mathrm{Ch}$. d. Ae. blieb lange Jahre das populärste Werk über Geburtshülfe und trug zur Erhöhung seines ärztlichen Ansehens wesentlich bei. So that denn auch Dr. Peter Ch. keine Fehlbitte, als er bei dem Könige (Carl II.) darum nachsuchte, $\mathrm{Hugh}$ als seinen ältesten Sohn gleichfalls zum Leibarzt zu ernennen. Die Einsetzung in diese bei den Chamberlens nachgerade erblich gewordene Würde erfolgte denn auch October 1673.

Einige zerstreute Notizen zeitgenössischer Werke lassen schliessen, dass $\mathrm{Hugh} \mathrm{Ch}$. d. Ae. eine Zeit lang Friedensrichter war und sich auch in der Politik bemerklich machte. Er gehörte der Whig-Partei an. So grosses Glück sein Uebersetzungswerk machte, so übel erging es Hugh mit einer 1685 erschienenen eigenen Abhandlung, betitelt: ,Manuale medicum, oder: Kleiner Tractat der Arzneikunst im Allgemeinen, wie der Brechmittel und des Jesuitenpulvers ( $=$ Chinapulver) im Besonderen, von $\mathrm{Hugh} \mathrm{Chamberlain}^{1}$ ), Leibarzt seiner hochseligen Majestät

1) Bemerkenswerther Weise schrieb Hugh $\mathrm{Ch}$. d. Ae. seinen Namen hier nicht in der gewöhnlichen Weise, nahm auch nicht den Titel M. D. (Med. 
und Mitglied der Königlichen Gesellschaft zu London." Diese Schrift, eine Anpreisung aller Schrecken der Medicina crudelis, besonders aber des Brechens; welches mit dem Gebäracte verglichen wird und alles Schädliche aus dem Körper ,obenheraus“ treibe, war in so herausforderndem Tone gegen die Londoner Aerzte, insbesondere die Mitglieder des College of Physicians abgefasst, dass dieses energisch gegen den Autor vorging. Er wurde aufgefordert, sich dem College behufs Ablegung eines Examens und Erwerbung der Licenz zur Ausübung der inneren Heilkunde zu stellen, erschien aber nicht. Ein zur Kenntniss des College gekommener Todesfall in seiner Praxis gab bald Gelegenheit, ihn zur Verantwortung zu ziehen. Er hatte einer im sechsten Monat Schwangeren, die wahrscheinlich an einer Pneumonie erkrankt war, binnen neun Tagen vier Brechmittel, vier Abführmittel, ein Mittel, welches Mundgeschwüre und Speichelfluss bewirkte, gegeben und ihr dazu noch drei Mal zur Ader gelassen, bis sie nach Eintritt der Fehlgeburt starb. Hu gh fand es nun doch gerathener, vor dem College, welches die Sache ganz wie ein ordentliches Gericht (Comitia censoria) mit Zeugen verhandelte, zu erscheinen und eine Vertheidigungsschrift einzureichen, welche verworfen wurde. Er wurde der "Mala praxis" für schuldig befunden und zu 10 Pf. Sterl. Geldstrafe verurtheilt. Vor einer Einsperrung im Newgate-Gefängniss und gerichtlicher Ahndung rettete ihn nur eine weitere Busse von $10 \mathrm{Pf}$. Sterl. Dieser Zwischenfall schadete ihm durchaus nicht in seinem Ansehen. Namentlich blieb er bei Hofe in Gunst. So sollte er der Königin bei der Geburt des nachmaligen „Prätendenten" (Jacob III. Stuart) beistehen, kam aber zu spät, erst etwa eine Stunde nach Geburt des Kindes. Von den zahlreichen Feinden des verhassten Königs Jacob II. wurde dasselbe für untergeschoben erklärt. Ein späterer Brief Hugh Ch.'s d. Ae. an die Prinzessin Sophia (Sophia Dorothea, Kurprinzessin von Hannover, damals im Haag), welche ihn nach den Umständen bei der Entbindung der Königin hatte befragen lassen, wurde von den Historikern als wichtiges Beweismittel für die Rechtmässigkeit der Geburt Jacob III. angesehen. Hugh Ch. d. Ae. schildert unter Berufung auf seine Zugehörigkeit zur Partei der Whigs genau den Hergang und widerlegte

Doctor) an. Zum Mitgliede der Royal Society wurde er am 2. November 1687 gewählt. In dem Journalbuche derselben ist er ein Mial als „Dr. Hugh Chamberlain", ein Mal als "Chamberlane" aufgeführt. 
jene Gerüchte. Jacob II. berief sogar, als er zu St. Germain in der Verbannung lebte, Hugh Ch. zur Entbindung seiner Gemahlin dorthin (1692). Er musste aber in London bleiben, da er keinen Pass erhielt. Noch eine andere Dame aus dem königlichen Hause, Prinzessin Anna von Dänemark, entband Hugh Ch. von einem todten Prinzen und empfing 100 Guineen für seine Mühewaltung.

Im Jahre 1694 veröffentlichte Hugh Ch. d. Ae. ein weiteres medicinisches Werk unter dem langathmigen Titel: „Einige Fragen zur praktischen Heilkunde mit Bemerkungen hierüber zu Jedermanns ernstlicher Erwägung bescheiden vorgetragen in Hinsicht besserer Bewahrung von Leben und Gesundheit (welche so nothwendig ist und Jedem theuer sein sollte) von. H. Chamberlen ${ }^{1}$ ), ordentlicher Leibarzt des hochseligen Königs Carl II." Diese Schrift, nichts als eine erweiterte Auflage seines ,Manuale medicum“, enthielt zum Schluss einen Entwurf ,zur Neuordnung der Heilkunde", welchen er 1689 gemacht hatte und dem Parlamente einreichen wollte. Es scheint aber nicht geschehen zu sein. Damit schliesst die medicinische Thätigkeit Hugh Ch.'s d. Ae., soweit sie in die Oeffentlichkeit trat, ab und beginnt eine Laufbahn ganz im Style seines Vaters.

Sehon lange hatte er sich mit dem Plane getragen, eine Landbank zu gründen, d. h. eine Bank mit dem Rechte, Noten resp. Papiergeld gegen hypothekarische Sicherstellung von ländlichem Grundeigenthum auszugeben gemäss dem Satze, dass Jedermann das Recht haben sollte, soviel Papiergeld zu verausgaben, als sein Besitzthum werth sei. Dieses Papiergeld sollte auch als allgemeines Zahlungsmittel Geltung haben. Er veröffentlichte aber seine Ideen hierzu erst 1690 unter dem Titel: „Dr. Hugh Chamberlen's Vorschlag, England reich und glïcklich zu machen." Er versprach goldene Berge, ganz wie sein Vater in der Broschüre: „Des armen Mannes Anwalt oder Englands Samariter."

Es konnte nicht verfehlen, dass ein solches gleissendes Project nicht nur die Menge, sondern sogar das Parlament bestach dessen beide Häuser December 1693 die Landbank concessionirten. Macaulay geht in seiner Geschichte Englands sowohl mit den chimärischen, vom volkswirthschaftlichen Standpunkte grund-

1) Hier ist der Name in der. gewöhnlichen Weise geschrieben, olne Beifügung der Titel M. D. und F. R. S. 
falschen Lehren Chamberlen's wio mit der Verblendung des Parlaments scharf ins Gericht.

Wie sehr Chamberlen's Unternehmen damals das Publikum erregte, zeigt die Flut von Schriften für und wider, ernst und spöttisch, welche sich damit beschäftigten.

Aveling führt deren allein 45 an, welche zum grössten Theile $\mathrm{Hugh} \mathrm{Ch}$. selbst, sowie seinen Theilhaber Briscoe zum Verfasser hatten. Für zehn Jahre seines Lebens war $\mathrm{Hugh} \mathrm{Ch}$. ganz von diesem Finanzunternehmen in Beschlag genommen, dessen Vortheile er schriftlich nicht nur, auch mündlich in öffentlichen Vorträgen mit einer Nachhaltigkeit und Geschicklichkeit verfocht, die einer besseren Sache würdig waren.

Der Zusammenbruch der ,lange gehätschelten Landbank“" konnte nicht ausbleiben, und $\mathrm{Hugh} \mathrm{Ch}$. ging daraus nicht mit reinen Händen hervor. Sandford, ein zeitgenössischer Chronist, berichtet darüber wie folgt: ,1699. Dienstag, 21. März. Dr: Cham berlain, die Mann-Hebamme, der alleinige Anzettler und Leiter der Landbank, hat sich Schulden halber nach Holland zurückgezogen." Hugh $\mathrm{Ch}$. kehrte nicht wieder nach England zurück, doch kann er sich, entgegen der gewöhnlichen Annahme, nicht unmittelbar nach Holland gewendet haben, sondern begab sich erst nach Schottland, um sein Bankproject auch vor das dortige Parlament zu bringen. Ein weiterer Beweis seines Aufenthaltes in Schottland ist die 1702 erschienene Schrift: „Die grossen Vortheile einer Vereinigung der beiden Königreiche von Schottland und England." Hierin zeigt er so umfassende Kenntnisse der Verhältnisse, wie sie nur im Lande selbst erworben sein konnten. Es ist sehr bemerkenswerth, dass dieses letzte aller von den. Chamberlens aufgestellten Projecte das einzige sein sollte, welches zur Verwirklichung kam. Ob Hugh Ch. d. Ae. die Vereinigung Schottlands mit England, 12. Mai 1707, erlebte, weiss man nicht, doch steht Aveling nicht an zu rühmen, dass seine Schrift, welche die mancherlei Vortheile derselben in bündiger und logischer Darstellung dem Publikum auseinandersetzte, auf ihr Zustandekommen von grossem Einfluss gewesen sei.

Wann $\mathrm{Hugh} \mathrm{Ch.} \mathrm{d.} \mathrm{Ae.} \mathrm{nach} \mathrm{Holland} \mathrm{übersiedelte;} \mathrm{ist} \mathrm{un-}$ bekannt; man weiss nur, dass er in Amsterdam noch einige Jahre prakticirte, wo er die Bekanntschaft von Roonhuysen machte. Hier ist er wahrscheinlich auch gestorben. Aveling spricht die Hoffnung aus, dass es holländischen Geburtshelfern, welche sich 
für die Sache interessirten, gelingen werde, in den Archiven des Landes Documente ans Licht zu fördern, welche über die letzte Lebenszeit des merkwürdigen Mannes Aufschluss zu geben geeignet seien.

Bezeichnender Weise dürfen wir als die eigentliche Ursache der Uebersiedelung von $\mathrm{Hugh} \mathrm{Ch}$. d. Ae. nach Holland wohl auch als die Triebfeder seines berüchtigten Handels mit Roonhuysen eben den Bankerott jenes Landbankunternehmens betrachten, und ist es nicht ausgeschlossen, dass die Noth ihn gezwungen hat, das lange gehütete Geheimniss der Familie gegen Geld preiszugeben.

Dr. Paul Chamberlen war der zweite Sohn von Dr. Peter Chamberlen und am 22. October 1635 in London geboren. Von seinem Vorleben ist nichts bekannt, ebensowenig, auf welcher Universität er sich den Doctorgrad erworben haben soll. Er prakticirte in London als „Mann-Hebamme“, wie aus der Andeutung seines Bruders zu entnehmen ist, im Besitze des Familiengeheimnisses. Er suchte es diesem auch als Finanzmann gleichzuthun, wie aus einem an das Parlament gerichteten Gesuche hervorgeht, worin er sich anheischig macht, der Regierung zinslos Capitalien $z u$ verschaffen, die Steuereinkünfte zu vermehren u. dgl. m. Er wartete vergeblich darauf, dass sich das Parlament seine Recepte geben liess. Jenes plumpe Gesuch weist uns aber den Schlüssel zu seinem Charakter. Er war ein Charlatan und Quacksalber, indem er als solcher ,ein schmerzstillendes Halsband für zahnende Kinder und kreissende Weiber" vertrieb. Aus dem ,Daily Journal" ist uns eine Reclame für dasselbe erhalten, ganz im Stilo derjenigen der Jetztzeit.

Dr. Paul Chamberlen starb 1717.

Dr. John Chamberlen war der vierte Sohn von Dr. Peter Chamberlen und gemeinsam mit seinen Brüdern $\mathrm{Hugh}$ und Paul im Besitze des Familiengeheimnisses, welches er als prakticirender Geburtshelfer verwerthete. Er scheint Doctor der $\mathrm{Me}-$ dicin gewesen zu sein, doch weiss man nicht, welche Universität ihm den Grad verliehen hat. Man kennt den Zeitpunkt der Abfassung und Beglaubigung seines Testamentes, doch nicht den seines Todes.

Hugh Chamberlen der Jüngere, geboren 1664, war der älteste Sohn von $\mathrm{Hugh} \mathrm{Chamberlen} \mathrm{senior} \mathrm{(siehe} \mathrm{Abbildung}$ S. 135, nach dem Marmor-Kenotaph in der Westminster-Abtei). 
Wie Peter Ch. d. Ae. seinem Sohne Peter eine bessere Erziehung und Ausbildung angedeihen liess, als ihm selbst zu Theil geworden war, so that es auch $\mathrm{Hugh} \mathrm{Ch}$. d. Ae. mit seinem Sohne Hugh Ch. d. J. Er brachte ihn in das Trinity college zu Cambridge, wo er 1683 zum Magister artium graduirt wurde. Noch vorher verfasste er in Anlass der Vermählung der Prinzessin Anna mit Prinz Georg von Dänemark ein schwungrolles lateinisches Hochzeitsgedicht.

Am 30. October $1684 \mathrm{kam}$ Hugh Ch. jun. nach Leyden und begann hier das Studium der Medicin. Am 8. October 1689

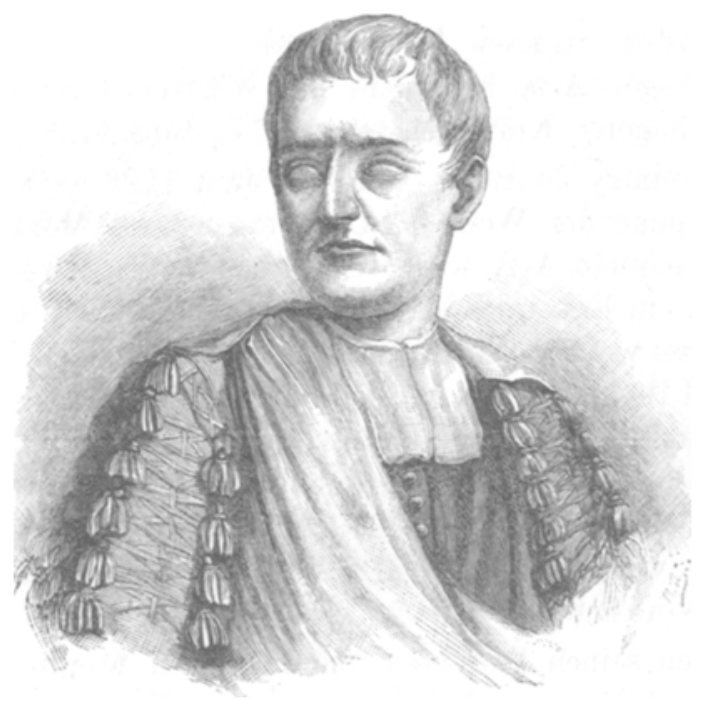

wurde er in Cambridge zum Doctor promovirt Am 2. April 1694 ward er nach Ablegung der vorgeschriebenen Prïungen in das College of Physicians, London, als Mitglied aufgenommen.

Als das College bald darauf sich an die Spitze eines wohlthätigen Unternehmens stellte zu dem Zwecke, armen Kranken unentgeltlich ärztlichen Rath und billige Arznei zu verschaffen, lehnten sich die Apotheker und ein Theil der von diesen geschürten Mitglieder des College selbst dagegen auf. Hugh Ch. jun. finden wir aber mit unter den eifrigsten Stützen der menschenfreundlichen Bewegung, die er auch durch einen ansehnlichen Geldbeitrag unterstützte. Zum Beweise der hohen Achtung, in welcher er bei seinen Fachgenossen stand, kann dienen, dass er 
utm 30. September 1707, dann in der Folge noch zwei Mal, 1719 und 1721, zu der ehrenvollen Würde eines Censor des College erwählt wurde. Krankheitshalber legte er dann diese Stelle nieder.

„Hugh Chamberlen des Jüngeren Leben verlief im Vergleiche zu dem seines Vaters und Grossvaters ruhig und ereignisslos. Er war weder Enthusiast, sei es auf religiösem oder politischem Gebiete - seinem gesellschaftlichen Umgange nach war er Tory und Jacobit -, noch starrte er voller Projecte für seine und Anderer Wohlfahrt. Infolge dessen liegt auch nur spärlicher Stoff zu seiner Biographie vor."

In den letzten zehn Jahren seines Lebens wohnte er in einer der vornehmsten Strassen Londons als gesuchter Geburtshelfer und zurerlässiger Arzt besonders der höheren Gesellschaft. Er starb nach längerer Kränklichleit am 17. Juni 1728.

Das „Country Journal“ vom 22. Juni 1728 verkündet sein Ableben in folgender Weise: „Der vergangenen Montag gestorbene ausgezeichnete Arzt und Geburtshelfer Dr. $\mathrm{Hugh} \mathrm{Cham-}$ berlen war ein Enkel des berühmten Dr. Peter Chamberlen, welcher ebenso wie sein Vater und seine Onkel dem König Jacob I., Karl I., Karl II., Jacob II., Wilhelm nebst ihren Gemahlinnen, sowie der Königin Anna als Leibärzte dienten. Er war der Letzte dieser alten Familie, welche im Königreich die Geburtshülfe ausübte, ausgenommen Dr. W al ker ${ }^{\mathbf{l}}$ ) in der grossen Suffolk-Strasse, welcher (von weiblicher Seite) gleichfalls ein Enkel des erwähnten Dr. Peter Chamberlen ist."

Von allen seinen Patienten und Freunden wandte ihm Niemand höhere Achtung und Gunst zu als der Herzog und die Herzogin von Buckinham. Nach dem Tode des Herzogs wurde er der intimste Vertraute seiner Wittwe und blieb stets in ihrer Umgebung. Ja, der Tod ereilte ihn gerade in Buckinham House, in welchem er bis zum Begräbniss aufgebahrt blieb. Die Herzogin errichtete ihm auch jenes prachtvolle Grabdenkmal in der Westminster-Abtei, welches ärztliche Besucher gemeiniglich zu der Meinung verleitet, es gelte ,dem Erfinder der Geburtszange“",

1) Nach Dr. William Douglas war dieser Dr. Walker der Erfinder des englischen Zangenschlosses, wie aus einem von Jenem an Smellie gerichteten Briefe ${ }_{*}^{*}$ (a Letter to Dr. Smellie etc., 1748, S. 8) zu entnehmen ist, worin es heisst: "Dr. Walker giebt vor, Dr. Chamberlen's Forceps verbessert zu haben, aber in Wirklichkeit hat er ihn verdorben, indem er ihn "männlich" und "weiblich" gemacht hat." 
während es doch nur dem Letzten der. Chamberlens als Zeichen persönlicher Hochschätzung und Anhänglichkeit von den Buckinhams gestiftet wurde. Die lateinische Grabschrift ist von Bischof Atterbury, mit welchem $\mathrm{Hugh} \mathrm{Ch}$. eng befreundet war, verfasst.

Mit Hugh Chamberlen junior stirbt die Familie im Mannsstamme aus. Seine drei Töchter heiratheten sämmtlich hochangesehene Männer und pflanzten in weiblicher Linie die Familie noch einige Generationen hindurch fort. In dem ruhigen, edlen und tadellosen Lebensgange des Letzten der Chamberlens liegt ein versöhnender $\mathrm{Zug}$, und ist es gewiss von Bedeutung, dass die Lüftung des Geheimnisses der Familie in die letzten Lebensjahre $\mathrm{Hugh} \mathrm{Ch.} \mathrm{d.} \mathrm{J.} \mathrm{fällt,} \mathrm{wenn} \mathrm{auch} \mathrm{directe} \mathrm{Nachrichten} \mathrm{dar-}$ über fehlen, dass er selbst es war, welcher den Schleier hob.

Nach documentarischer Schilderung des Lebens der Chamberlens geht Aveling über zu einer

\section{Kritischen Erörterung der Hauptfrage nach dem Erfinder der Geburtszange.}

Die Neuheit und Vorzüglichkeit der Chamberlen'schen Geburtszange gegenüber den älteren, äusserst unvollkommenen Instrumenten der Araber, des $\mathrm{Ru}$ eff u. A., bestand darín, dass sie aus zwei trennbaren Theilen zusammengesetzt war. Da ausgemachter Weise nur einem Gliede der vier Generationen dieser Geburtshelfer-Familie die Ehre der Erfindung gebührt und es nicht nöthig ist, die Ansprüche Anderer hierauf noch besonders zurückzuweisen, so bleibt blos die eine Frage zu lösen: „Welcher von den Chamberlens war der Erfinder?" Um diese Frage befriedigend $\mathrm{zu}$ beantworten, wird es erforderlich sein, mit der Zeit zu beginnen, wo die Bekanntschaft mit der Zange allgemein wurde, und dann zu versuchen, die Spuren der Erfindung des Instrumentes rückwärts zu verfolgen bis zum Erfinder.

Das Geheimniss der Chamberlens wurde nicht vor dem Beginn des 18. Jahrhunderts gelüftet. Die erste Beschreibung der Geburtszange stammt von Chapman 1733 (d. i. fünf Jahre nach Hugh Ch.'s Tode). Er schrieb damals: ,ich muss bemerken, dass es sehr verschiedeneArten von Forceps giebt, welche keineswegs alle gleichmässig brauchbar sind, weshalb auf ihre Form sehr zu achten ist.“ Dann fügt er hinzu: „Das von Dr. Chamberlen angedeutete Geheimniss, wodurch sein Vater, 
zwei Brüder und er selbst solche Kinder rettete, welche sich in Kopflage zur Geburt stellten, jedoch nicht durch natürliche Wehen geboren werden konnten, war, wie allgemein angenommen wird und keinem Streit mehr unterliegt, der Gebrauch des Forceps, welcher jetzt allen hervorragenden Fachleuten in Stadt und Land wohl bekannt ist."

Wie lange Zeit vor 1733 mag nun das Geheimniss schon offenbart gewesen sein? Chapman war Landarzt in einem kleinen Orte in Essex und hatte nicht so leicht Gelegenheit wie seine Londoner Collegen, neue Erfindungen in seinem Fache alsbald kennen za lernen; gleichwohl muss er geraume Zeit, bevor er seine Beschreibung veröffentlichte, sich des Forceps bedient haben, da er äussert: ,mein Forceps ist vor vielen Jahren aus so weichem Metall gefertigt worden, dass er sich bog oder nachgab, oder Aenderung in seiner Krümmung erlitt. Er war wie üblich so gebaut, dass die Schraube an dem einen Theil oder der einen Seite desselben befestigt war. Ich bediente mich dessen einige Jahre; da es mir aber oft begegnete, dass das Instrument seitwärts abglitt, so bekam ich eine so geringe Meinung von demselben, dass ich es nur selten, ja in einem Zeitraume von zehn Jahren nicht ein einziges Mal gebrauchte."

Es ist nicht unwahrscheinlich, dass auch William Sormon (Verfasser von „English Midwife“, 1671) über die Zange Einiges wusste, da er in dem Kapitel über die Zeichen des Todes der Frucht und die Mittel, diese auszuziehen, Folgendes schreibt: ,wenn aber keine von all diesen Arzneien anschlägt, so giebt es noch einige andere, jedoch ernstere und gewaltsamere Verfahren, nämlich die Ausziehung mit dem Haken, Zangen (tongs) und anderen Instrumenten." Ganz fest steht, dass Bau und Gebrauch der Geburtszange während der ersten Hälfte des 18. Jahrhunderts nach und nach allgemein bekannt wurde, so dass Fielding 0 ut 1742 schreiben konnte (,A Treatise of Midwifery", p. 153. Dublin): ,das verwendbarste Instrument ist der lange Forceps, welcher jetzt in Europa überall in Gebrauch ist."

Die A uffindung der Chamberlen'schen Instrumente im Jahre 1813 in einem geheimen Verschlage der von Dr. Peter $\mathrm{Ch}$. angekauften Besitzung Woodham Mortimer Hall bei Maldon, Essex, welche bis 1715 im Besitze der Familie verblieb, wird von Aveling nach einem späteren Berichte eines in Maldon prakticirenden Dr. Robert Lee (s. Medico-chirurgical Transactions, 
XXVII, 1861) genauer und etwas abweichend von der Darstellung geschildert, die Dr. H. H. Carwardine in seinem, die Uebergabe der Instrumente begleitenden Berichte der Königl. medicinischen und chirurgischen Gesellschaft zu London davon gegeben hatte (Medico-chirurgical Transactions, IX, 1818). Dr. Lee bezog seine Auskünfte über die näheren Umstände des Fundes von einer Frau Codd, welche damals Woodham Mortimer Hall bewohnte. Die eigentliche Entdeckerin des Versteckos, deren Namen wohl verdient, der Nachwelt ibermittelt zu werden, war deren Mutter, eine Mrs. Kemball.

Diese Instrumente, welche in den Räumen der Medicinischen und chirurgischen Gesellschaft in einem Glaskasten sorgsam aufbewahrt werden, sind:

3 gefensterte Hebel; der eine derselben läuft in einen kurzen stu mpfen Haken aus, der zweite endigt als scharfer Haken, der dritte hat fast die Form eines Zangenblattes, zeigt aber keinen Schlitz für eine Achse und endigt als scharfer Haken.

3 scharfe Haken, von denen 2 am anderen abgebogenen Ende stumpf auslaufen, während der dritte einen flachen, herzförmigen Handgriff aufweist.

3 Schling en (fillets), 2 aus Fischbein mit Atlasband, eine 3. aus Fischbein und schwarzer Seide, an jedem Ende durchbohrt von je 2 Schnürlöchern.

4 Zangen. Fig. 1 eine Zange von sehr roher Bauart, das eine Blatt 121/2" $(31,25 \mathrm{~cm})$, das andere $13^{\prime \prime}(32,5 \mathrm{~cm})$ lang;

Fig. 1.

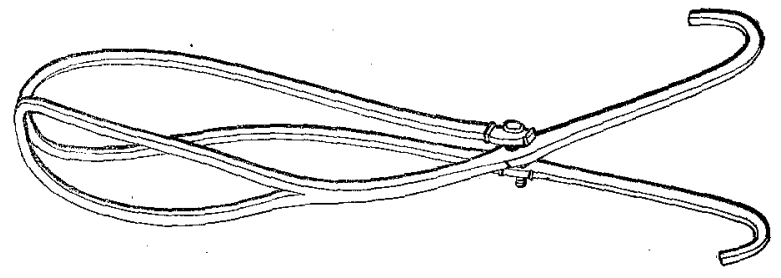

Länge dès Blattes bis zum Schlosse beiderseits 8 " (20 cm); Länge des Fensters in dem einen $5^{\prime \prime}(12,5 \mathrm{~cm})$, in dem anderen $8^{\prime \prime}$ $(20 \mathrm{~cm})$. Der eine Griff hat eine Länge von $41 / 2 "(11,25 \mathrm{~cm})$, der andere eine solche von 4 " $(10 \mathrm{~cm})$; beide enden nach aussen umgebogen als stumpfe Haken. Die beiden Hälften des Instrumentes sind durch eine Schraube mit einander verbunden. Der Schrauben- 
kopf ist oval, glatt und ohne den iiblichen Einschnitt geformt. Die Spitzen der Blätter berühren einander nach Schluss des Instrumentes. Dieses Instrument ist zweifellos die erste Geburtszange, von welcher alle übrigen jetzt in Gebrauch befindlichen Zangenformen abstammen.

Fig. 2. Zange von $12^{\prime \prime}(30 \mathrm{~cm})$ Länge; Länge bis zum Schloss $91_{12}^{\prime \prime} "(23,75 \mathrm{~cm})$; Länge des Fensters in dem einen Blatte

Fig. 2.

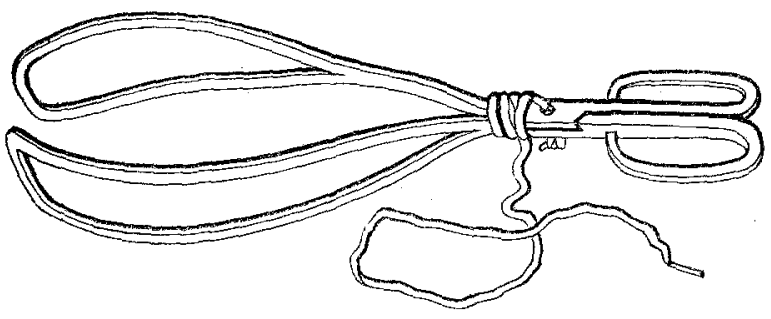

$81 / 2 "(20 \mathrm{~cm})$, in dem anderen $51 / 4^{\prime \prime}(13,12 \mathrm{~cm})$; Breite des Fensters an dem ersteren $11_{4}{ }^{\prime \prime}(3,12 \mathrm{~cm})$, an dem anderen $1{ }^{1 / 8} "$ $(2,8 \mathrm{~cm})$. Die Griffe sind $3 \frac{1}{4} "(8,12 \mathrm{~cm})$ lang und in Gestalt einer Oese gebogen, welche auf der einen Seite zwei Fingern, auf der anderen dem Daumen bequem Platz gewährt. Die beiden Hälften des Instrumentes sind durch ein geflochtenes Band vereinigt, welches an dem einen Ende einen Knoten, an dem anderen einen Stift aufweist. Das Band wurde durch die Löcher, welche sonst zur Aufnahme der Schraube dienten, durchgesteckt und gestattete so dem Operateur, die Blätter beliebig zu offnen und zu schliessen.

Fig. 3. Zange von 12 “ $(18 \mathrm{~cm})$ Länge, ähnlich in der BauFig. 3.

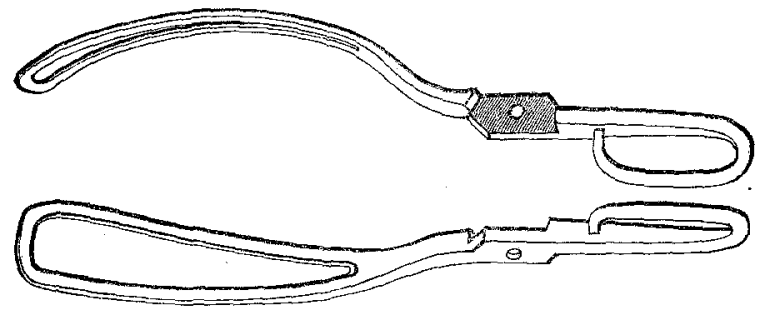

art wie die vorige, nur dass die Fenster gleich gross sind, 6" $(15 \mathrm{~cm})$ lang und 1 “ $(2,5 \mathrm{~cm})$ breit. 
Fig. 4. Kräftige Zange, $13^{\prime \prime}(32,5 \mathrm{~cm})$ lang; Länge der Blätter bis zum Schloss 8“ $(12 \mathrm{~cm})$; Länge des Fensters $5^{\prime \prime}$ $(12,5 \mathrm{~cm})$, Breite $1^{\prime \prime}(2,5 \mathrm{~cm})$. Die Griffe sind ösenförmig und $5 “(12,5 \mathrm{~cm})$ lang. Die Vereinigung der beiden Zangenhälften

Fig. 4.

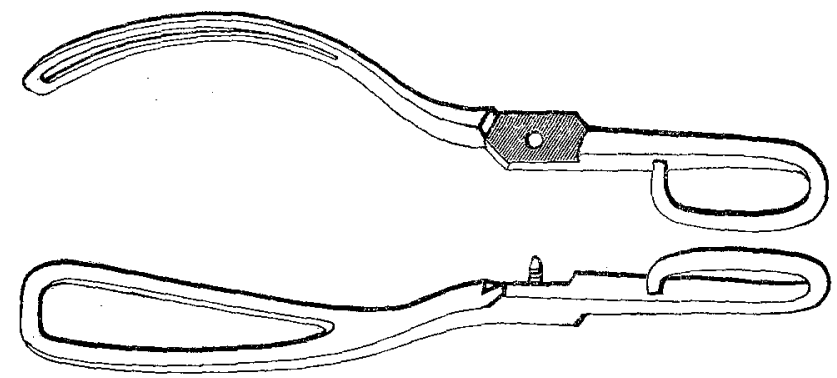

geschah durch einen festen Stift an dem einen Blatte, welcher lose in einen runden Ausschnitt des anderen hineinpasste. Die Spitzen der Blätter stehen nach Schlass des Instrumentes 11/4" $(3,12 \mathrm{~cm})$ von einander $a b .1)$

Wie man, gewiss überraschender Weise, sieht, zeigen die Modelle Fig. 1 und 4 schon ganz ein Grundmerkmal der späteren L e ret'schen Zangen, ein Achsenschloss.

Als Erfinder des "englischen Schlosses" wurde früher schon Walker genannt. Ein Vorläufer desselben, doch mehr improvisirter Weise, scheint $\mathrm{Chapman}$ gewesen zu sein: er verlor die Schraube seiner Zange und fand, dass das Instrument seinen Dienst ohne solche besser versah als mit.

Die wichtige Entdeckung dieser Instrumente in dem Hause von Dr. Peter Chamberlen schliesst ohne Weiteres die beiden letzten Generationen der Familie als Bewerber um die Erfinderschaft derselben aus. $\mathrm{Hugh} \mathrm{Ch}$. senior und junior, Paul und John Ch. waren zweifellos im Besitze des Familiengeheimnisses, aber Keiner von ihnen hat Anspruch auf die Ehre, die Geburtszange erfunden zu haben. Die z. Zt. allgemein verbreitete Annahme, dass Dr. Peter $\mathrm{Ch}$. der Erfinder sei, ist irrthümlich, denn es geht aus Allem, was über ihn berichtet wurde, unwiderleglich hervor, dass er selbst, ebenso wie seine Nachkommen von

1) Man wird an diesen Abbildungen durch Vergleich mit denjenigen unserer gebräuchlichen Lehrbücher leicht erkennen, dass die Chamberlen'schen Zangen vielfach falsch wiedergegeben sind. 
ihm, die Kenntniss der Geburtszange von seinem Vater übermittelt bekam.

Prüft man die Beschreibung der in Dr. Peter Ch.'s Haus aufgefundenen Instrumente, so springt eine wichtige und auffallende Thatsache in die Augen, nämlich, dass jede Gattung derselben in drei Exemplaren vertreten ist. Allerdings fanden sich vier Zangen, doch ist die eine derselben von so roher Bauart, dass sie kaum gebraucht worden sein konnte. Dieses bemerkenswerthe Zusammentreffen drängt mit Nothwendigkeit zu der Annahme, dass die aufgefundenen Instrumente nicht nur Dr. Peter $\mathrm{Ch}$. angehört haben können, sondern auch seinem Vater und Onkel, da das Geheimniss wohl schwerlich hätte gewahrt werden können, wenn die Instrumente der beiden Letzteren nach deren Tode in andere Hände fielen, als die von Dr. Peter Ch. Dieser führte in der ersten Zeit seiner ärztlichen Laufbahn ein lustiges und ausschweifendes Leben, Noth und Bedrückung, wie sie den Geist schärft, die Erfindungsgabe weckt, hatte er nicht zu erdulden gehabt. Wir wissen ausserdem (siehe S. 124), dass er schon in jungen Jahren in den Besitz des Familiengeheimnisses kam und frühzeitig eine überlegene Stellung als Geburtshelfer erlangte, wodurch sich die Auflehnung der Hebammen gegen ihn, ,der nicht mehr Bart habe als sie selbst“, erklärt. Allerdings befasste er sich vielfach mit mechanischen Entwürfen, bekannte aber einerseits selbst, dass sie nicht von eigenen Erfindungen herrührten, andererseits trugen diese so sehr den Charakter des Chimärischen an sich, dass man ihn für alles eher, denn für einen vernünftigen und praktischen Mechaniker halten kann. Ferner sagt $\mathrm{Hugh} \mathrm{Ch.} \mathrm{d.} \mathrm{A} \mathrm{e,} \mathrm{in} \mathrm{der} \mathrm{Vorrede} \mathrm{zu} \mathrm{seiner} \mathrm{Uebersetzung} \mathrm{des}$ Mauriceau: ,Mein Vater, meine Brüder und ich selbst sind auf einen Weg gelangt (attained to), Frauen zu entbinden u. s. w." Er bedient sich hier aber nicht des Ausdruckes ,erfunden" (invented), wie er wohl gethan haben würde, wenn sein Vater wirklich der Erfinder war. Dass er dies aber ganz sicher nicht war, lässt sich eben aus Dr. Peter Ch.'s Schriften selbst nachweisen, nämlich aus einer Stelle der "Stimme in Rhama“, wo es heisst: „Mein Ruhrn zog mir Neider und heimliche Feinde zu, deren Zahl wuchs, als mich mein Vater in die Entbindungskunst eingeweiht hatte."

Es geht auch aus den Aufzeichnungen der Jahrbücher des „Königl. Collegium der Aerzte von London“ hervor, dass Dr.Peter's 
Vater und Onkel (d. i. die Brüder Peter d. A e. und Peter d. J.) im Besitze ganz besonderer Geschicklichkeit und Mittel zur Behandlung schwieriger Geburten gewesen sind, was den Mitgliedern des Collegium zu grossem Verdrusse gereichte und Veranlassung wurde zu jenem Einschreiten gegen Peter $\mathrm{Ch}$. d. J., welches früher (siehe S. 123) geschildert wurde. Was das für eine geheime Methode war, deren sich die beiden Brüder bedienten, kann darüber noch ein Zweifel obwalten? Keinesfalls würden sie die hervorragende Stellung errungen haben, welche sie wirklich einnahmen, wenn sie sich nicht in ihrem Fache durch besondere Geschicklichkeit auszeichneten. Wie Vieles stand ihrem Emporkommen nicht im Wege?! Als Fremde sah man sie misstrauisch und gehässig an, als Flüchtlinge waren sie selbst arm und niedergedrückt. Gewandtheit, Fleiss und Thatkraft lehrte sie alle im Wege stehenden Schwierigkeiten zu überwinden, und so sehen wir einen Peter Ch. d. Ae. ungeachtet aller dieser Hemmnisse dahin gelangen, selbst die Königin mehrmals zu entbinden, sehen wir beide Brüder mächtige Freunde gewinnen, sich zu ehrenvollen Stellungen aufschwingen und ein bedeutendes Vermögen sammeln.

Endlich bliebe dann nur noch die eine Frage zu entscheiden, welcher von den beiden Brüdern (Peter d. Ae. und Peter d. J.) der Erfinder der Geburtszange sei. Da giebt denn eine bisher übersehene Stelle aus der Einleitung zu Smellie's Lehrbuch der Geburtshülfe Aufschluss, soweit dies bei derartiger Beweisfiuhrung möglich ist. Indem Smellie von dem Instrumente spricht, dessen sich die Chamberlens bedient hätten, bemerkt er: „und heisst es, dass es von dem Onkel erfunden worden ist" (,and said to be contrived by the uncle"). Unter ,dem Onkel" kann Niemand sonst verstanden werden als Peter Chamberlen der Aeltere, da Dr. Peter Chamberlen keinen als Geburtshelfer prakticirenden Bruder besass. So kann denn mit fast absoluter Bestimmtheit Peter Chamberlen der Aeltere') als derjenige bezeichnet werden, welchem die Ehre der Erfindung der Geburtszange wirklich gebührt.

Da wohl als sicher angenommen werden darf, dass man schwerlich je dahin gelangen wird, ein anderes und reicheres Ma-

1) Budin feiert (1. c.) diese Thatsache in folgender Weise: "Chamberlen, inventeur du forceps, est né à Paris; Levret, qui l'a modifié si heureusement, était à Paris; Tarnier, qui l'a perfectionné, babite aussi Paris." 
terial zur Lösung der gestellten Frage beizubringen, als es $A v e=$ ling gelungen ist, so darf man gewiss mit Fug behaupten, er habe nicht nur die lang umstrittene Controverse nach dem wahren Erfuder der Zange gelöst, sondern auch die Gesehichte der Chamberlen'schen Familie in einer Weise aufgehellt, berichtigt und erweitert, dass eine an sich wenig wahrscheinliche Erschliessung etwa unbekannter, neuer Quellen daran wenig ändern würde. Damit hat diese lange umstrittene Frage einen Abschluss gefunden, bei welchem es vielleicht für immer verbleiben wird. 